\title{
Mäßiger Alkoholkonsum vermindert Gewichtszunahme nach der Menopause
}

\section{Viele Frauen nehmen während und nach den Wechseljahren an Gewicht zu. Nun wurde gezeigt, dass die Ge- wichtszunahme auch vom Alkohol- konsum abhängt.}

— Die „Women's Health Initiative“-Studie stellte mit 15920 Probanden, die älter als 50 Jahre waren und sieben Jahre lang beobachtet wurden, die Datenbasis. Aufgenommen wurden Frauen mit normalem Gewicht (BMI 18,5- $<25 \mathrm{~kg} /$ $\mathrm{m}^{2}$ ). Ein Drittel der Frauen gab an, kei-

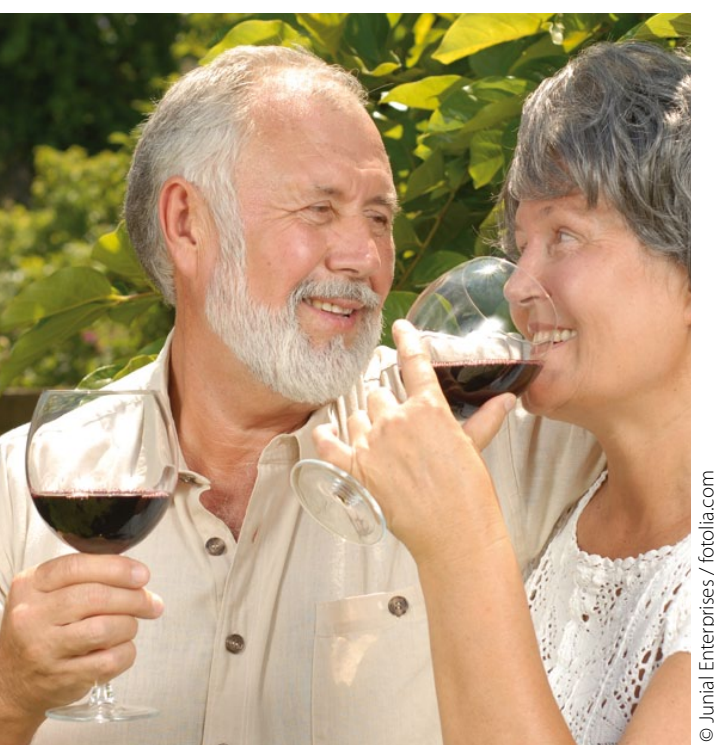

Macht mäßiger Alkoholkonsum eine schlanke Figur? nen Alkohol zu trinken. Frauen mit einem Alkoholkonsum in der obersten Quintile hatten ein um 35\% geringeres Risiko übergewichtig und ein um $88 \%$ geringeres Risiko, adipös zu werden, als alkoholabstinente Frauen. Die Gewichtszunahmen in Abhängigkeit vom Alkoholkonsum waren wie folgt:

- $<0,2 \mathrm{~g} / \mathrm{d}:+1,4 \mathrm{~kg}$

- $0,2-1,8 \mathrm{~g} / \mathrm{d}:+1,4 \mathrm{~kg}$

- 1,8-6,5 g/d: $+1,0 \mathrm{~kg}$

- 6,5-13,6 g/d: $+0,6 \mathrm{~kg}$

- 11,6-197 g/d: +0,2 kg.
Eine geringere Gewichtszunahme ging u.a. auf das Konto von Wein (-25\%), höherprozentigen Alkoholika $(-15 \%)$ und Bier $(-10 \%)$. Der Alkoholeffekt war bei Frauen über 60 Jahren ähnlich wie bei solchen zwischen 55 und 60 Jahren.

- C. A. Thomson et al.

Alcohol consumption and body weight change in postmenopausal women: results from the Women's Health Initiative. Int J Obes. 2012;36:1158-1164

\section{Kommentar}

Das Ergebnis verblüfft zunächst, da Alkohol mit $7 \mathrm{kcal} / \mathrm{g}$ einen beträchtlichen Energiegehalt hat. Er liegt niedriger als der von Fett $(9$ $\mathrm{kcal} / \mathrm{g}$ ), aber höher als der von Kohlenhydraten und Proteinen (4 kcal/g). Dennoch war das Resultat nicht ganz unerwartet, gab es doch vor einigen Jahren schon kleinere Studien mit ähnlichen Ergebnissen. In der jetzigen Auswertung konnte eine Dosis-Wirkungs-Beziehung zwischen dem Alkoholkonsum und der Gewichtsänderung festgestellt werden. Wie kann man sich das vermeintliche $\mathrm{Pa}$ radox erklären? Zum einen stellt Alkohol die Gefäße weit und erwärmt den Körper, was mit Energieverbrauch einhergeht. Zum anderen wird Alkohol über einen anderen Stoffwechselweg (mikrosomal) abgebaut, was mit einer geringeren ATP-Produktion ein- hergeht. Schließlich verursacht Alkohol eine Stimulation der sympathischen Aktivität. Alle Mechanismen erklären, weshalb Alkohol den Energieverbrauch erhöht.

In der Studie hat die überwiegende Zahl der Frauen wenig bzw. sehr wenig Alkohol getrunken. Nur $17 \%$ tranken mehr als $14 \mathrm{~g} / \mathrm{d}$. Das Ergebnis soll keinesfalls zu vermehrtem Alkoholkonsum ermutigen, denn bekannt ist auch, dass hoher Alkoholkonsum die Gewichtszunahme steigert und insbesondere die Entwicklung einer abdominalen Adipositas begünstigt. Möglicherweise ist das bezüglich der Gewichtszunahme beobachtete Phänomen ähnlich dem bei koronarer Herzkrankheit. Auch dort reduzieren mäßige Alkoholmengen das Krankheitsrisiko, größere jedoch erhöhen es.

A. WIRTH = 